Report WD 87-07

On An Off-Shore Pipe Laying Problem

R.M.M. Mattheij

S.W. Rienstra

August 1987

Wiskundige Dienstverlening Faculteit der Wiskunde en Natuurwetenschappen Katholieke Universiteit Toernooiveld 6525 ED Nijmegen The Netherlands 


\title{
ON AN OFF-SHORE PIPE LAYING PROBLEM
}

\author{
R.M.M. Mattheij S.W. Rienstra
}

The final version of this paper will be submitted for publication elsewhere 


\begin{abstract}
In this paper we consider a model for determining the shape of a pipe laid by a barge. We investigate how the solution of a resulting second order non-linear differential equation depends critically on the (unknown) vertical bottom reaction force; by this we can explain some difficulties met when approximating the solution numerically. An important part of the analysis is based on studying a pendulum analogy in a time dependent gravity field, by which we obtain existence and uniqueness results.
\end{abstract}

\title{
1. Introduction
}

The transportation of gas or oil from offshore wells requires the installation of pipelines to connect the well to some mainland station. Typically such pipes are $16-36$ inch in diameter and may be up to several hundreds of kilometers long. Usually these pipes are laid in relatively shallow ( $\sim 50 \mathrm{~m})$ and deep $(\sim 300 \mathrm{~m})$ water, although recently technology has been developed to handle very deep water (300-1000 $m$ and more). Part of the technology is in the material of which the pipes are being made (high quality steel), but very much depends on the laying technique too. The main problem to be dealt with is buckling due to too high bending stresses during laying; pipe collapse due to a too high water pressure becomes also important at greater depths, but we shall not consider that problem here.

At present the pipes are being laid using a so called laybarge where the pipe sections are welded together, and then gradually released off the barge to the sea bed. If the welding ramp is horizontally positioned, the pipe is released via a (circular) stinger. According to the shape of the suspended pipeline this technique is called $S$-lay. If the welding ramp is in vertical position the technique is called $J$-lay. Here we shall concentrate (although not in principle) on the $S$-lay technique, which is the usual one for not very deep water. The analysis we give below is equally applicable for $J$-lay configurations though.

Usually, the pipes are relatively thin-walled and, without additional weight, would float on the water. This weight is provided by application of a concrete coating, giving the pipes a net specific gravity of about 1.05 to 1.30 times that of water. (The value depends on the product to be transported, and the 
presence of sea currents.) This non-negligible own weight, especially in deep water, tends to give the pipe during laying a shape with such a high curvature, that without precautions the pipe would buckle. Therefore, the laybarge is equipped with tension machines to apply a certain horizontal tension, just sufficient to stretch the $S$ curve and reduce the bending stresses to a safe level.

The practical question now is: given a certain geometry, how much tension is needed and, furthermore, which is the optimal position of the stringer. On the one hand, repairing a buckled pipe is very expensive, but on the other hand, so are the tension machines and other equipment, so rather accurate calculations are required. Given the large number of parameters involved this problem has to be solved again for each new case, and a mathematical model including a computer program is obviously required (Ref. $1,2,3,4,5,6,7,8,9)$. This will be the subject of the present paper.

The problem as such arose from requests from offshore companies, who were interested in a routine efficient and fast enough for a small computer, to be used on board in order to be able to respond immediately to unforeseen variations in (e.g.) steel quality, concrete quality, or other problem parameters. In trying to develop a program we hit severe numerical problems. In particular, since the problem is nonlinear, we were often not able to make the Newton method involved converge. In search for the phenomena behind this problem we encountered some interesting mathematical questions. An explanation of the aforementioned problem was the presence of a 'close' family of other solutions. We shall treat this problem to some extent as we believe that it nicely demonstrates why numerical black boxes should be treated with great care, also in industrial problems; another reason is that the fairly messy nonlinear structure is elucidated substantially by invoking a mechanical analogy (\$3). The existence of the desired solution as a limiting case of the family of close solutions is treated in $\$ 4$. Finally, in $\$ 5$ we consider some more mathematical questions, arsising as mere off shoots, in particular concerning the bending energy.

\section{The model}

In this section we shall derive a model for the problem outlined above and indicate some questions related to this problem. In figure 1 we have sketched the configuration of a laybarge. The pipe is guided 
into the water by a stinger having a certain uniform (adjustable) radius of curvature $R$. Typically three forces are exerted on the pipe: a) gravity, due to the not negligible net weight, b) a horizontal tension $H$, applied by the tension machines mounted on the vessel which is supposedly anchored, and c) the (unknown) vertical bottom reaction force $V$.

By denoting:

$$
\begin{aligned}
& s=\text { arclength } \\
& Q=\text { effective pipe weight per unit length } \\
& M=\text { bending moment } \\
& E I=\text { flexural rigidity }
\end{aligned}
$$

we have (Fig.2; cf. ref. 10,11)

$$
M=E I \frac{d \psi}{d s} \quad(\text { Bernoulli }- \text { Euler law })
$$

$$
\frac{d M}{d s}=H \sin \psi-V_{s} \cos \psi \quad \text { (equilibrium of moments) }
$$

$$
\frac{d H}{d s}=0 \quad \text { (horizontal tension remains constant) }
$$

$$
\frac{d V_{s}}{d s}=Q
$$

From (2.2) we derive the basic equation

$$
E I \frac{d^{2} \psi}{d s^{2}}=H \sin \psi-(Q s-V) \cos \psi
$$

where we note that

$$
x(s)=\int_{0}^{s} \cos \psi d s^{\prime}, y(s)=\int_{0}^{s} \sin \psi d s^{\prime}
$$

Besides $\psi$ and $V$, also the free length $L$ (from bottom to stinger departure point) is unknown. This requires four boundary conditions in total to be specified. The ones we will adopt here are not exactly corresponding to the configuration as drawn in fig.1 (which would include a free boundary at the 
stinger, cf. ref.8), but somewhat simplified, however, without altering the characteristics of the problems discussed.

We will consider in principle:

$$
\begin{gathered}
\psi(0)=0, \frac{d \psi(0)}{d s}=0 \quad \text { (free end supported by the bottom) } \\
\frac{d \psi(L)}{d s}=-\frac{1}{R} \quad \text { (stinger curvature) } \\
\int_{0}^{L} \sin \psi(s) d s=D \quad \text { (the total depth) }
\end{gathered}
$$

We now like to find the shape of the pipe, given a horizontal force $H$. This force is needed to give the pipe a low enough curvature to prevent it from buckling. This buckling usually happens when the combined stress due to bending, tension, and water pressure exceeds the yield stress, i.e., the maximal stress that the steel can afford without plastic deformation.

We first reduce the problem parameters to a basic set. Introduce dimensionless quantities

$$
t=s / D_{0}, \quad l=L / D_{0}, \quad d=D / D_{0}
$$

$$
h=H D_{0}^{2} / E I, q=Q D_{0}^{3} / E I, \quad v=V D_{0}^{2} / E I
$$

For convenience we introduced an unspecified length $D_{0}$, in order to prevent limiting cases as $Q=0$, $H=0, L \rightarrow \infty, D \rightarrow \infty$ to produce artificially singular, and therefore less interpretable problems. So in general the number of dimensionless parameters may be reduced by one, and we may assume, for example, $h=1$ or $q=1$. So we obtain

$$
\ddot{\psi}=h \sin \psi-(q t-v) \cos \psi
$$

with boundary conditions

$$
\psi(0)=0, \quad \dot{\psi}(0)=0
$$




$$
\begin{aligned}
& \dot{\psi}(l)<0 \text { given } \\
& \int_{0}^{1} \sin \psi d t=d
\end{aligned}
$$

By augmenting (2.6a) with

$$
\begin{aligned}
& \dot{v}=0 \\
& i=0
\end{aligned}
$$

we have a fourth order ODE (2.6) with four BC (2.7). When this system is 'fed' into a BVP routine on a computer, one needs to specify initial estimates for $\psi, \dot{\psi}, v$, and $l$, as one has to solve by iteration, say using Newton's method. For problems with values of $l \approx d$ this turns out to be increasingly and, eventually, prohibitively difficult. In order to produce a reliable program we therefore investigate what phenomenon is possibly causing these troubles. First, we note that for longer pipes there will be a considerable part with negligible bending stress where the pipe behaves like a catenary $(\ddot{\psi} \approx 0$; cf. ref. 8). Only at the ends, in boundary layers, the $\ddot{\psi}$-term is of importance. If $l$ is large enough, the pipe will be, in the catenary part, nearly vertical (i.e. $\psi \approx \pi / 2$ ). Obviously, the entire solution can be built up by singular perturbation techniques (e.g. ref. 8 ). Our interest here, however, concentrates on what may happen in the first part where $\psi$ grows from 0 to $\pi / 2$; in particular for which values of $v$ this vertical shape (for $/$ large) is obtained. Therefore we derive the following subproblem:

Find in the family of solutions of $(2.6 \mathrm{a})$ with $(2.7 \mathrm{a})$ the

(2.8) 'critical' $\psi(t, v)$ (with corresponding $v$ ) which satisfies

the condition $\psi \rightarrow \pi / 2$ for $t \rightarrow \infty$.

(For short, we will here and below always imply $\psi:=\psi(\bmod 2 \pi))$.

For convenience later we have denoted the dependence on $v$ explicitly. Since we have no dependence on $l$ any more, we have a third order problem now.

For the subsequent analysis, it is advantageous to introduce the first integral of (2.6a), which amounts to the elastic free energy density of the bent pipe (ref. 11)

$$
u(t)=\dot{\psi}^{2} / 2=h-h \cos \psi-(q t-v) \sin \psi+q \int_{0}^{t} \sin \psi d t^{\prime}
$$


The total energy is then

$$
E=\int_{0}^{\infty} u(t) d t
$$

Intuitively we may expect the problem (2.8) not to have a unique solution (and, likewise, also (2.6), (2.7)), as the pipe could possibly make several bends and loops. In fig. 3 we have given some indication of the intrinsical behaviour of $\psi$ (in cartesian coordinates) for various values of $v$. This observation is the clue to the numerical difficulties mentioned earlier: we shall show that the most natural solution of (2.8) (with a minimal energy $E$ ) is dense in a family of solutions which are, at least numerically, close to the required one of (2.6) with (2.7) if $d$ is large. This then explains why it is hard, sometimes impossible, to single out the desired one. To do this we shall employ a useful analogy which is introduced in the next section.

\section{Pendulum analogy}

The variety and character of the solutions of (2.6a) would be much easier predicted and classified if we can find an analogous problem, described by the same equation, but easier interpretable.

If $q=0$ such an analogy exists indeed, and is actually a classic result due to Kirchhoff (cf. ref.10). It consists of a mathematical pendulum in a gravity field $(-h, v)$, where $\psi$ now denotes the angle of the pendulum with the positive $y$-axis, and $t$ is time; (fig. 4a). For given $v, \psi$ will oscillate between $\psi=0$ and its mirror in the line $(-h, v): \psi=2 \pi-2 \operatorname{arctg}(v / h)$. If $v=0$ the pendulum will remain stationary in its (unstable) initial position $\psi=0$, as the oscillation period tends to infinity for $v \rightarrow 0$.

For $q \neq 0$ it is possible to generalise the pendulum analogy if we use a more general (admittedly more artificial) time dependent gravity field $(-h, v-q t)$; (fig. $4 \mathrm{~b})$. Then, as long as $v-q t>0$, the force field is directed to the right half (i.e. $x>0$ ), and $\psi$ will tend to return to the interval $(0, \pi)$; however, once $v-q t$ has changed sign and increases with $t$ without bound in negative direction, the force field points to the left, and, as time increases, forces $\psi$ to remain in a decreasing interval around $\psi=3 \pi / 2$; (fig. 5a). If $v$ is large, this process takes some time, and $\psi$ starts to oscillate around $\psi=\pi / 2$; (fig. 5b). 
In a similar fashion, $\psi$ starts to oscillate between 0 and $2 \pi$ if $h$ is large; however, if $q$ is large, $\psi$ is almost immediately forced to its final phase of oscillations around $\psi=3 \pi / 2$.

This behaviour is reflected in fig. 6 where several (numerical) solutions of eq. (2.6a) are plotted (in cartesian coordinates $(x, y))$. From these pictures it is immediately clear that the generalised pendulum analogy, supported by some continuity arguments, provides very simply the evidence of the existence of multiple solutions, particularly if $v$ is large enough.

Another interesting result that can be derived from the analogy is the nature of the critical solutions, with $\psi \rightarrow \pi / 2$ for $t \rightarrow \infty$. If $h=0, \psi=\pi / 2$ is clearly a solution, but an increasingly unstable one if $v-q t<0$. If $h \neq 0, \psi=\pi / 2$ is not a solution any more, although if $t \rightarrow \infty$ there may exist a (delicate) path for $\psi$ to approach $\pi / 2$ in such a way that the tangential component of the large force $(-h, v-q t)$ is never large enough to remove $\psi$ from $\pi / 2$. (The existence of such a solution is not trivial and we will return to this subject in the next section.) From the unstable nature of the solution $\psi \rightarrow \pi / 2$, with $v=v_{c}$ say, it is clear that near $v_{c}$ there are infinitely many $v$ with solutions that, after some time, will move away from the neighbourhood of $\pi / 2$, and will eventually approach $3 \pi / 2$ (though in an oscillatory way). Especially in a numerical contex this is important because it implies that for only very small variations in $v$ we may have several different solutions.

\section{Existence of the 'critical' solutions of (2.8)}

We now investigate the existence of the solution $\psi\left(t, v_{c}\right)$, where $v_{c}$ is such that we have the intuitively most likely solution of $(2.8)$, as sketched in fig. 7 . In the next section we shall show that this solution has minimal bending energy.

Two particular properties of the sought solution are

solution
(4.1a) $0<\psi\left(t, v_{e}\right)<\pi / 2$
$0<t<\infty$
(4.16) $0<\dot{\psi}\left(t, v_{c}\right)$
$0<t<\infty$ 
In the original problem setting, we considered $v$ as a parameter to be determined along with the solution from an ODE formulation (via $\dot{v}=0$ ). Here we shall look for such values of $v$ that the ODE (2.6a) with (2.7a) has a solution with the properties (4.1) by fine tuning $v$. Inspired by (4.1), let us define two sets of $v$-values giving rise to two families of solutions $\psi$ of (2.6a), (2.7a)

$$
\begin{aligned}
& V_{1}=\left\{v>0 \mid\left(\exists t_{1}<\infty\right)\left(\dot{\psi}\left(t_{1}, v\right)=0\right) \wedge\left(\psi(t, v)<\pi / 2,0<t<t_{1}\right)\right\} \\
& V_{2}=\left\{v>0 \mid\left(\exists t_{2}<\infty\right)\left(\psi\left(t_{2}, v\right)=\pi / 2\right) \wedge\left(\dot{\psi}(t, v)>0,0<t<t_{2}\right)\right\}
\end{aligned}
$$

The sets $V_{1}$ and $V_{2}$ are constructed such that either $(4.1 \mathrm{a})$ or $(4.1 \mathrm{~b})$ does not hold.

Property 4.3. (i) $V_{1} \cap V_{2}=\emptyset$, (ii) $v_{c} \in \mathbb{R}_{+} \backslash\left(V_{1} \cup V_{2}\right)$

Proof. (i) Suppose $v \in V_{1} \cap V_{2}$, then we obviously have $t_{2}=t_{1}$. Hence $\psi\left(t_{2}, v\right)=\pi / 2, \dot{\psi}\left(t_{2}, v\right)=0$. From (2.6a) this implies that $\ddot{\psi}\left(t_{2}, v\right)=h$. If $h=0$ then all derivatives of $\psi$ vanish, so $\psi$ is constant, which is a contradiction. If $h>0, \psi$ must have a (local) minimum at $t=t_{2}$, implying $\dot{\psi}(t, v)<0$ for $t<t_{2}$. This, however, would only occur if $v \notin V_{2}$, so the result follows by contradiction.

(ii) Let $v>0, v \notin V_{1} \cup V_{2}$, then

$$
[\forall t\{(\dot{\psi} \neq 0) \vee(\exists \tau<t \text { with } \psi(\tau)=\pi / 2)\}] \wedge[\forall t\{(\psi \neq \pi / 2) \vee(\exists r<t \text { with } \dot{\psi}(\tau)=0)\}]
$$

However, both for $t_{2}<t_{1}$ and $t_{1}<t_{2}$ the requirements $\dot{\psi}\left(t_{1}\right)=0$ and $\psi\left(t_{2}\right)=\pi / 2$ are mutually exclusive. So (4.1) implies that $\psi$ converges as $t \rightarrow \infty$ to, say, a constant $c<\pi / 2$. Then $\ddot{\psi} \rightarrow-(q t-v) \cos c \neq 0$, which give a contradiction. Hence $\psi \rightarrow \pi / 2$ for $t \rightarrow \infty$ if $v$ is element of the complement of $V_{1} \cup V_{2}$ (which, however, may be empty).

We now finally arive at

Theorem 4.4. $V_{1}, V_{2}$, and the complement of $V_{1} \cup V_{2}$ are not empty; moreover $\sup \left(V_{1}\right)=v_{c}=\inf \left(V_{2}\right)$.

Proof. (a) Let $v$ be small (i.e. close to zero) and consider $\psi$ for $t$ small. Then we may linearize (2.6a) to obtain

$$
\ddot{\psi}=h \psi-q t+v
$$


For this elementary equation it easily follows that for some small value of $t$, say $t=t_{1}$, we have $\dot{\psi}(t, v)=0$. By choosing $v$ small enough, $\psi$ can be kept as small as we like along $\left[0, t_{1}\right]$; in any case small enough for the linearization, and smaller than $\pi / 2$, which thus yields a nonempty $V_{1}$.

(b) To prove that $V_{2}$ is nonempty, we look for large enough $v$. To start, we observe that for $t \rightarrow 0$ such that $\psi \rightarrow 0$ we have $\psi \simeq \frac{1}{2} v t^{2}$. So for $v \rightarrow \infty$ we have $\psi=2 / v$ at $t=2 / v$. Furthermore, using (2.9), we have along $0<t<v / q$ (as long as $\psi<\pi$ ) $\frac{1}{2} \dot{\psi}^{2}>0$, so $\dot{\psi}>0$, and $\psi$ increases. Again using (2.9), we thus obtain for $2 / v<t<3 v / 4 q$ (and, of course, $\psi$ not close to $\pi) \frac{1}{2} \dot{\psi}^{2}>(v-q t) \sin \psi>(v-q t) 2 / v>\frac{1}{2}$. So $\dot{\psi}>1, \psi=\int_{0}^{1} \dot{\psi} d t>t$, and therefore $\psi$ can be made arbitrarily large (at least larger than $\pi / 2$ ) before $\dot{\psi}$ vanishes.

(c) From the foregoing and property 4.3 we conclude that $\sup \left(V_{1}\right)=v_{c}=\inf \left(V_{2}\right)$ as follows. First, we observe that if a $v_{c}$ exists, it is a single point: consider a solution $\psi=\psi_{c}+\phi, v=v_{c}+\epsilon$, in the neighbourhood of the critical solution ( $\phi$ and $\epsilon$ small). Then for large enough $t$, when $\psi_{c} \rightarrow \pi / 2$, we have

$$
\ddot{\phi}=\left(q t-v_{c}\right) \phi
$$

with exponentially growing solutions. So near $v_{c}$ there can be no other critical $v$ 's.

Finally, only the existence of $v_{c}$ remains to be shown. For this, we consider a sequence of angles, increasing to $\pi / 2$. With each angle we select a corresponding solution $\psi_{n}$ of which $\dot{\psi}_{n}$ vanishes for the first time at this angle (at time $t=t_{1, n}$, say). So by definition, the corresponding sequence $\left\{v_{n}\right\}$ is a subset of $V_{1}$. Since $\ddot{\psi}\left(t_{1, n}\right)<0$ we have with (2.6a)

$$
q t_{1}>v+h \operatorname{tg} \psi\left(t_{1, n}\right)
$$

so $t_{1, n} \rightarrow \infty$, and we have thus constructed a sequence in $V_{1}$ converging to $v_{c}$. $\quad$

\section{Further investigations of the equation (2.8)}

It is interesting to note that we should expect a sequence of critical $v$ values, of which $v_{c}$, considered in the previous section is just the first member. Intuitively this follows immediately from the pendulum analogy as mentioned in $\$ 3$. Here we like to look for a more precise (though qualitative) characterization. 
Before we do that we remark that the existence of another such 'critical v' will complicate the numerical problem, as sketced in $\$ 2$, even more; for if we might zoom in on that wrong value, during some iterative procedure (say due to a completely off-value initial estimate for $v$ ), we not only encounter the troubles associated with so many close solutions, but also the fact that our solution is completely wrong, if it converges at all.

Let us recall the energy density relation (2.9). We can characterise a solution $\psi(t, v)$ by its energy

$$
E_{l}(v)=\int_{0}^{l} \frac{1}{2} \dot{\psi}^{2}(\tau, v) d \tau=\frac{1}{2} \int_{0}^{\psi(l, v)} \dot{\psi}(\tau, v) d \psi
$$

In our problemsetting we are interested more particularly in the case $l \rightarrow \infty$ and the question therefore arises whether $E_{\infty}(v)$ exists. This question is addressed in the following two properties.

Property 5.2. $E_{\infty}\left(v_{c}\right)$ is finite.

Proof. If $t$ is sufficiently large, we have

$$
\psi\left(t, v_{c}\right)=\pi / 2-\operatorname{arctg} \frac{h}{q t-v_{e}} \simeq \pi / 2-h / q t
$$

So $\dot{\psi}\left(t, v_{c}\right)=h / q t^{2}+O\left(t^{-3}\right)$, i.e. $\left[\dot{\psi}\left(t, v_{c}\right)\right]^{2}=O\left(t^{-4}\right)$. Hence $\frac{1}{2} \int_{0}^{l} \dot{\psi}^{2}\left(\tau, v_{c}\right) d \tau$ converges as $l \rightarrow \infty$. $\quad$ o

Property 5.3. If $\lim _{t \rightarrow \infty} \psi(t, v) \neq \pi / 2$ then $\lim _{t \rightarrow \infty} \psi(t, v)=3 \pi / 2$.

Moreover $\lim _{l \rightarrow \infty} E_{l}(v)$ does not exist.

Proof. As we have seen in $\S 3,3 \pi / 2$ is the only alternative attractor. So let $\psi(t)=[3 \pi / 2+\phi(t)] \bmod (2 \pi)$, then

$$
\ddot{\psi}=-q t \sin \phi=-q t \phi .
$$

The latter equation is therefore approximately equal to the Airy equation (cf. ref.12) with appropriate scaling of variable. So we can write

$$
\phi(t)=\alpha A i\left(-t / q^{\frac{1}{3}}\right)+\beta B i\left(-t / q^{\frac{1}{3}}\right),
$$

for some $\alpha, \beta \in \mathbb{R}$. Hence $\dot{\phi}(t)=\alpha \dot{A} i+\beta \dot{B} i \sim t^{\frac{1}{4}} \sin \left(t^{\frac{3}{2}}\right)$, so that $[\dot{\phi}(t)]^{2}=t^{\frac{2}{2}} \sin ^{2}\left(t^{\frac{3}{2}}\right)$. Since (with $\left.t=\tau^{\frac{3}{2}}\right)$

$$
\int_{\tau}^{T} t^{\frac{1}{2}} \sin ^{2}\left(t^{\frac{3}{2}}\right) d t=\int_{\tau^{\frac{3}{2}}}^{T^{\frac{3}{2}}} \sin ^{2}(r) d \tau
$$


we see that for $T \rightarrow \infty$ this integral and thus $E_{T}(v)$ (see 5.1)) diverges. $\diamond$

Theorem 5.4. Considered as a function of $v, E_{\infty}(v)$ has a local minimum at $v=v_{c}$.

Proof. (a) Denote by $\tilde{t}_{1}(v)$ the first time that $\ddot{\psi}=0$ (to become negative). Since $\ddot{\psi}_{c}$ starts positive and finally becomes negative, it follows that $T=\lim _{v \rightarrow v_{c}} \tilde{t}_{1}(v)<\infty$, and $\lim _{v \rightarrow v_{c}} \psi\left(\tilde{t}_{1}, v\right)<\pi / 2$. Hence there is a subset $\hat{V}_{2} \subset V_{2}$ with

$$
\ddot{\psi}\left(\tilde{t}_{1}\right)=0 \wedge \psi\left(\tilde{t}_{1}\right)<\pi / 2 \text { for } v \in \hat{V}_{2}
$$

Let $v \in \hat{V}_{2}$. Then by definition there is a time $t_{2}$ where $\psi=\pi / 2$. Here, $\ddot{\psi}=h>0$, so there is another time $\hat{t}_{2}$ with $\tilde{t}_{1}<\hat{t}_{2}<t_{2}$, where $\ddot{\psi}=0$ to become positive. Now denote by $\dot{\phi}(\psi, w)$ the moment (or 'speed') $\dot{\psi}$ as a function of the angle, and consider along $[0, \pi / 2]$ four sectors:

I: $\left[0, \psi\left(T, v_{c}\right)\right]\left(\ddot{\psi}\left(T, v_{e}\right)=0\right.$, force becomes negative after $t=T$ for $\left.v=v_{c}\right)$

II: $\left[\psi\left(T, v_{c}\right), \psi\left(\tilde{t}_{1}, v\right)\right]$

III: $\left[\psi\left(\tilde{t}_{1}, v\right), \psi\left(\hat{t}_{2}, v\right)\right] \quad\left(\ddot{\psi}\right.$ becomes positive after $\left.t=\hat{t}_{2}\right)$

IV: $\left[\psi\left(\hat{t}_{2}, v\right), \pi / 2\right]$

In I the 'force' $\ddot{\psi}$ is larger for the choice $v$ than for $v_{c}$. Hence with $v$ the point $\psi\left(T, v_{c}\right)$ is reached earlier and with a higher speed. In II this effect is reinforced as for the case $v_{c}$ the motion is slowed down in contrast to the case $v$. In III we have

$$
q t(v)<q t\left(v_{c}\right)
$$

so for any angle $\alpha \in \mathrm{III}, h \sin \alpha-(q t(v)-v) \cos \alpha>h \sin \alpha-\left(q t\left(v_{c}\right)-v_{c}\right) \cos \alpha$. Hence, despite the 'force' being negative, it is less negative for $v$ than for $v_{c}$.

Finally, in IV the motion for $v$ is accelerated again, in contrast to $v_{c}$. Summarising we thus have found: for any $\psi \in(0, \pi / 2)$ is $\dot{\phi}(\psi, v)>\dot{\phi}\left(\psi, v_{c}\right)$. From (5.1) we deduce

$$
E_{\infty}\left(v_{c}\right)<\frac{1}{2} \int_{0}^{\pi / 2} \dot{\phi}(\psi, v) d \psi<\lim _{l \rightarrow \infty} E_{l}(v)
$$

(b) Let $v \in V_{1}$ (see (4.1a)) be very close to $v_{c}$. At the backward swing the pendulum passes the point $\psi=0$ again, at time $t=t_{3}$, say. We have there $\ddot{\psi}\left(t_{3}, v\right)=-\left(q t_{3}-v\right)$ (which is of course $<0$ ). Now let the maximal 'force' $\ddot{\psi}$ at $\dot{\psi}\left(t, v_{c}\right)$ on $\left[0, \psi\left(T, v_{c}\right)\right]$ be $m$. Then it is possible by choosing $t_{3}$ large enough to 
make the 'force' $\ddot{\psi}$ at $\psi(\phi, v)$ on $\left[0,-\psi\left(T, v_{c}\right)\right]$ larger in absolute value than $m$ (by choosing $v$ sufficiently close to $\left.v_{c}\right)$; on $\left[-\psi\left(T, v_{c}\right),-\pi / 2\right]$ the force for $\psi$ is definite negative. We conclude from this that for such a choice of $v$ the 'speed' $\dot{\phi}$ on $[0,-\pi / 2]$ is larger in modulus than $\dot{\phi}\left(\psi, v_{c}\right)$ on $[0, \pi / 2]$, whence

$$
E_{\infty}\left(v_{\varepsilon}\right)<\frac{1}{2} \int_{0}^{-\pi / 2} \dot{\phi}(\psi, v) d \psi<\lim _{l \rightarrow \infty} E_{l}(v)
$$

From $\left({ }^{*}\right)$ and $\left({ }^{* *}\right)$ we deduce that $E_{\infty}\left(v_{c}\right)$ is a local minimum.

Remark. The proof for the case $v<v_{c}$ (part (b)) of the preceding theorem can also be based on employing the fact that for $v$ sufficiently close to $v_{c} \lim _{t \rightarrow \infty} \psi(t, v) \rightarrow-\pi / 2$ and using this fact in combination with Properties 5.2 and 5.3.

The question remains which of the solutions converging to $\pi / 2$ is the best. Apart from the solution $\psi\left(t, v_{c}\right)$ characterised by $(4.1)$ we may expect e.g. another solution $\psi\left(t, v_{c 2}\right)$ say with $v_{c 2}>v_{c}=: v_{c 1}$, having a terminal value $\lim _{t \rightarrow \infty} \psi\left(t, v_{c 2}\right)=5 \pi / 2$; i.e. this solution is still monotonic, passes $\pi / 2$ to come at rest only after completing another cycle. Similarly there are $v_{c i}$ to be expected such that $\lim _{t \rightarrow \infty} \psi\left(t, v_{c i}\right)=\pi / 2+(i-1) 2 \pi, \dot{\psi}(t)>0$. Existence will not be considered here, but basically we anticipate it to be similar as for the case of $v_{c 1}$. Also, for $v<v_{c 1}$ we may conjecture a sequence of ' $v$ values', such that after an initial interval $\dot{\psi}>0$, there is a point $t(v)$ where $\dot{\psi}(t(v))=0,0<\psi(t(v), v)<$ $\pi / 2$, and $\dot{\psi}<0$ for $t>t(v), \lim _{t \rightarrow \infty} \psi(t, v) \rightarrow-\pi / 2-2 k \pi$. It is interesting to realise, however, that whatever other critical value of $v$ may exist, $v_{c}=v_{c 1}$ is optimal in the sense of minimal energy, as is shown in

Theorem 5.5. $E_{\mathrm{o}}\left(v_{c}\right)$ is the global minimum.

Proof. $v_{e 2}$ should apparently be larger than $v_{c 1}$, as follows from the construction of the set $V_{2}$. If $v_{c 2} \in \hat{V}_{2}$ then it follows from part (a) of the proof of Theorem 5.4 that $\frac{1}{2} \int_{0}^{\pi / 2} \dot{\phi}\left(\psi, v_{c 2}\right) d \psi<E_{\infty}\left(v_{c}\right)$.

If $v_{c 2} \in V_{2} \backslash \hat{V}_{2}$ then it is simple to see that $\dot{\phi}\left(\psi, v_{c 2}\right)>\dot{\phi}\left(\psi, v_{c 1}\right)$ like in the aforementioned proof and thus that again $\frac{1}{2} \int_{0}^{\pi / 2} \dot{\phi}\left(\psi, v_{c 2}\right) d \psi<E_{\infty}\left(v_{c 1}\right)$. $。$ 


\section{Conclusion}

In this paper we have considered how the theoretical shape of a pipe depended on the value of the bottom reaction force $V$. The 'natural shape' for some critical value of $v=v_{c}$ for a long pipe was shown to have minimal bending energy. However, there are values of $v$ arbitrarily close to $v_{c}$, where this shape is completely different. This then explains (at least partially) why the nonlinear boundary value problem resulting from the pipe laying model is hard to solve without special techniques. A possible way out for this last problem is to use appropriate continuation techniques (e.g. in the length). Further investigations are currently under way.

\section{References}

1. D.A. Dixon, D.R. Rutledge, Stiffened Catenary Calculations in Pipeline Laying Problem. Journal of Engineering for Industry 90 (1), 1969, pp. 153-160.

2. J.T. Powers, L.D. Finn, Stress Analysis of Offshore Pipelines During Installation, Offshore Technology Conference 1071, 1969 .

3. J.R. Wilkins, Offshore Pipeline Stress Analysis, Offshore Technology Conference 1227, 1970.

4. G.C. Daley, Physical Interpretations of the Instabilities Encountered in the Deflection Equations of the Unconstrained Pipeline, Offshore Technology Conference 1933, 1974.

5. P. Terndrup Pedersen, Equilibrium of Offshore Cables and Pipelines During Laying, International Shipbuilding Progress, Vol.22, Dec. 1975, pp.399-408.

6. G. Maier, Optimization of Stinger Geometry for Deepsea Pipelaying, Journal Energy Resources Tech. 104, pp.294-301.

7. I. Konuk, Higher Order Approximations in Stress Analysis of Submarine Pipelines, ASME paper 80-PET-72, 1980.

8. S.W. Rienstra, Analytical Approximations For Offshore Pipelaying Problems, Proceedings of the First International Conference on Industrial and Applied Mathematics ICIAM 87, Paris 1987, Contributions from the Netherlands. (WI Tract 36, ed. by A.H.P. van der Burgh and R.M.M. Mattheij, 1987). 
9. M.J. Brown, L. Elliott, Pipelaying From a Barge, Mathematical Engineering For Industry 1 (1), 1987, pp.33-46.

10. R. Frisch-Fay, Flexible Bars, Butterworths, London 1962.

11. L.D. Landau, E.M. Lifshitz, Theory of Elasticity, Pergamon, Oxford, 1970.

12. M. Abramowitz, I.A. Stegun (eds.), Handbook of Mathematical Functions, National Bureau of Standards 1964.

R.M.M. Mattheij, Faculteit Wiskunde en Informatica, Technische Universiteit Eindhoven, Postbus 513, 5600 MB Eindhoven, The Netherlands.

S.W. Rienstra, Wiskundige Dienstverlening, Faculteit Wiskunde en Natuurwetenschappen, Katholieke Universiteit, Toernooiveld, 6525 ED Nijmegen, The Netherlands.

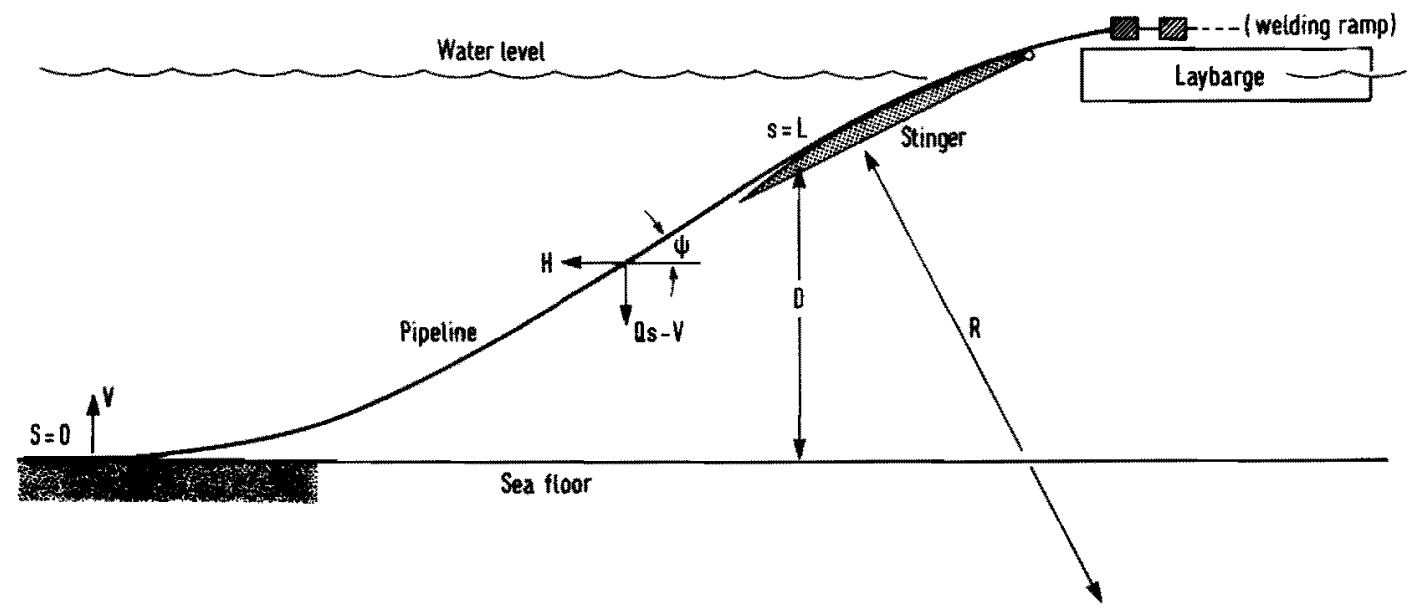

Figure 1. Sketch of the pipelay problem

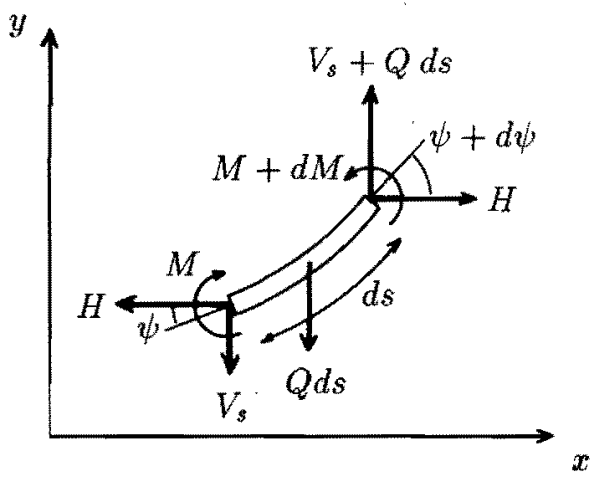

Figure 2 Equilibrium of pipe element 


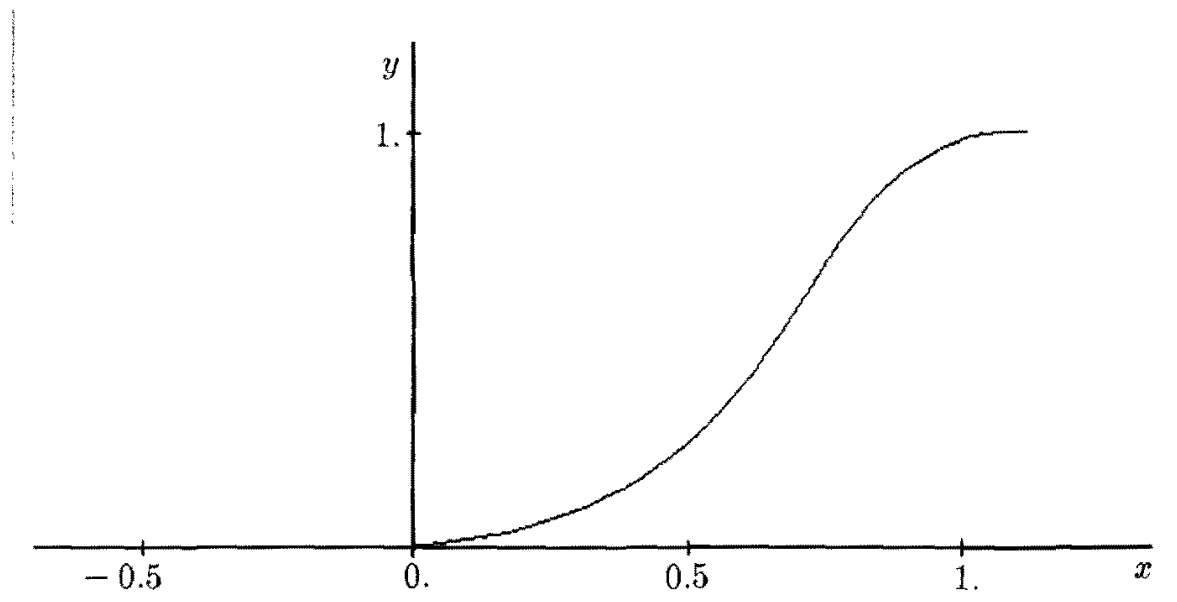

Figure 3a. $v=6.61, h=1, q=15$

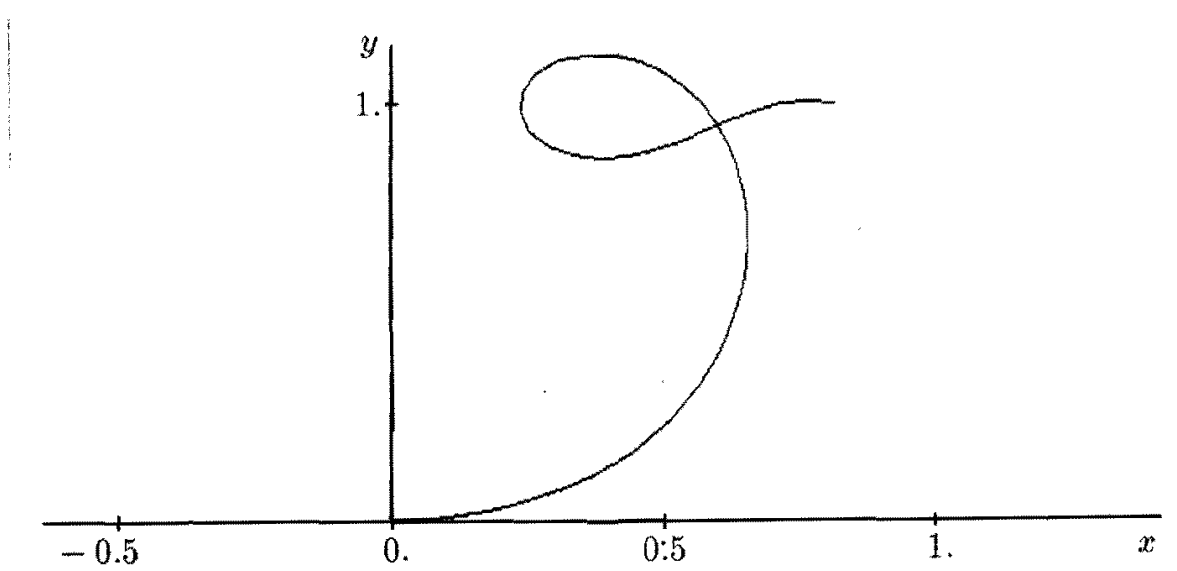

Figure 3c. $v=6.98, h=1, q=15$

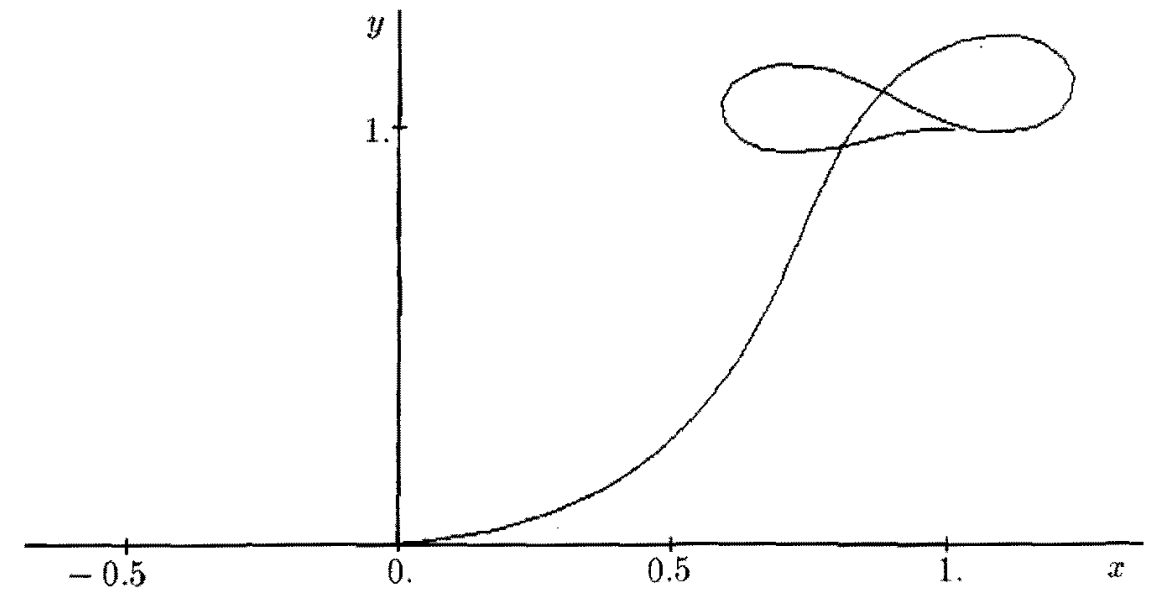

Figure 3b. $v=6.74, h=1, q=15$

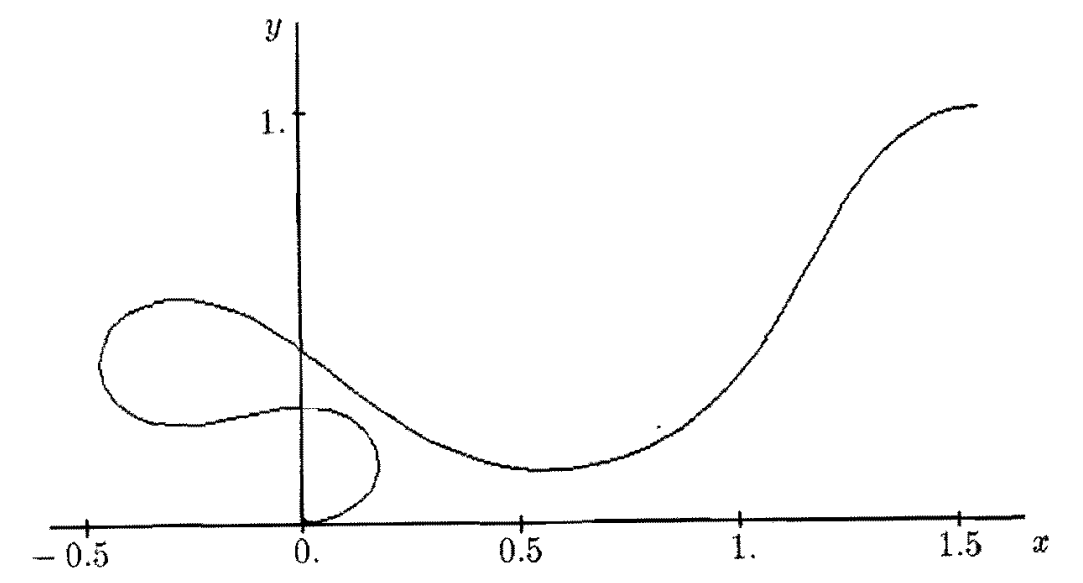

Figure 3d. $v=35.74, h=1, q=15$ 


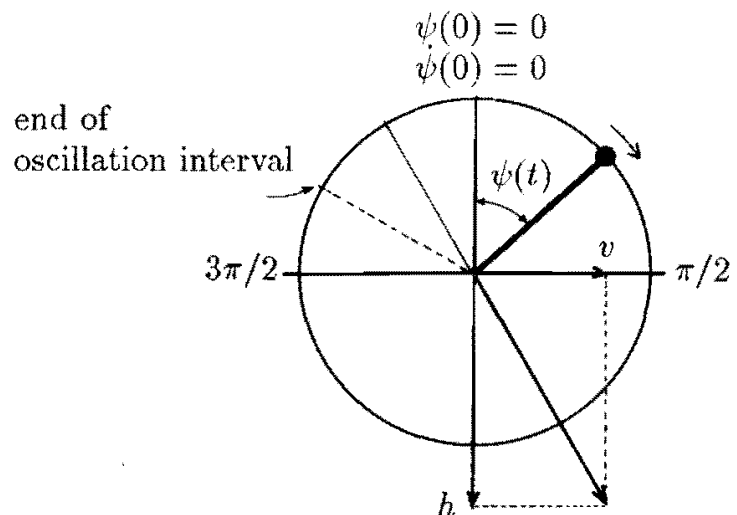

Figure 4a. Kirchhoff's pendulum analogy for $q=0$. Constant gravity field $(-h, v)$

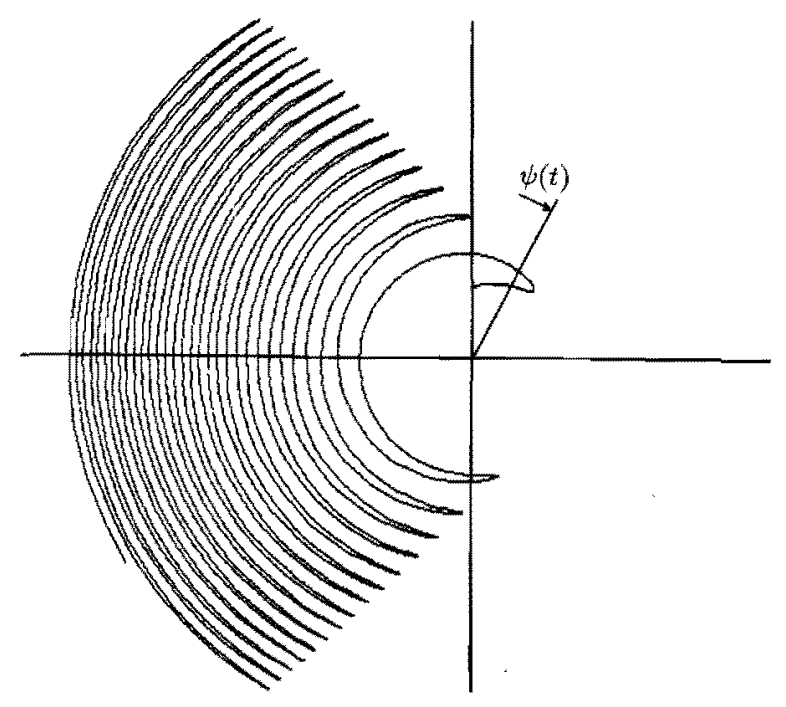

Figure 5a. Pendulum analogy (radial coordinate grows linearly in time) for $v=6, h=1, q=15$.

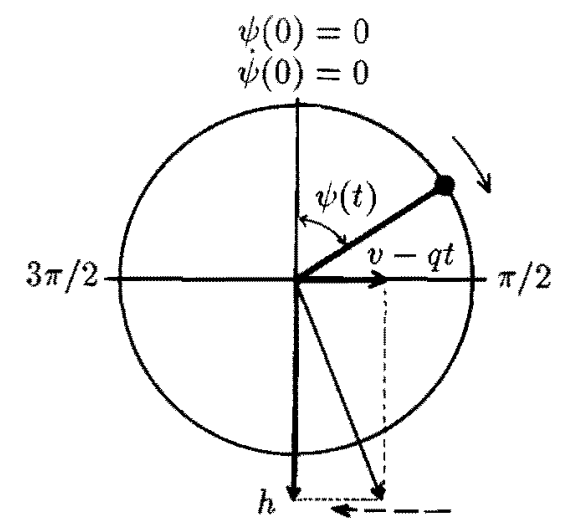

Figure 4b. Generalized pendulum analogy.

Time dependent gravity field $(-h, v-q t)$.

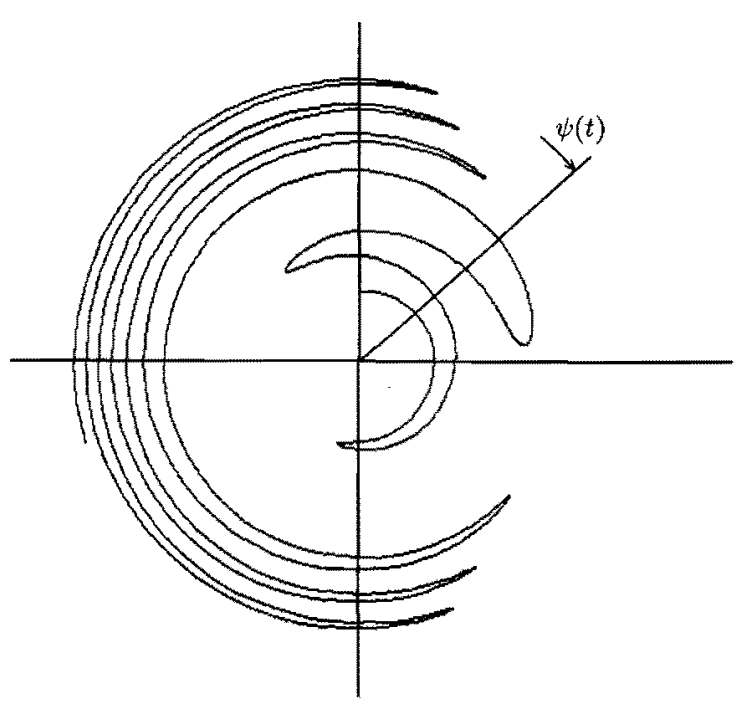

Figure 5b. $v=35.63, h=1, q=15$ 


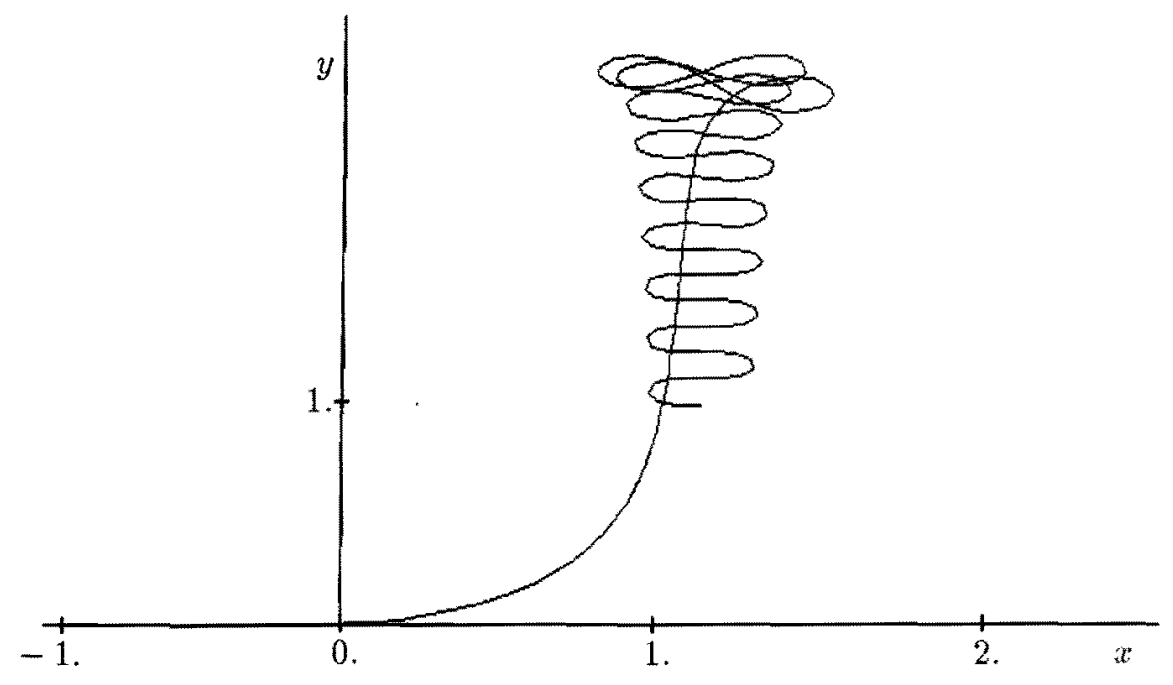

Figure 6a. $v=6.837, h=1, q=15$

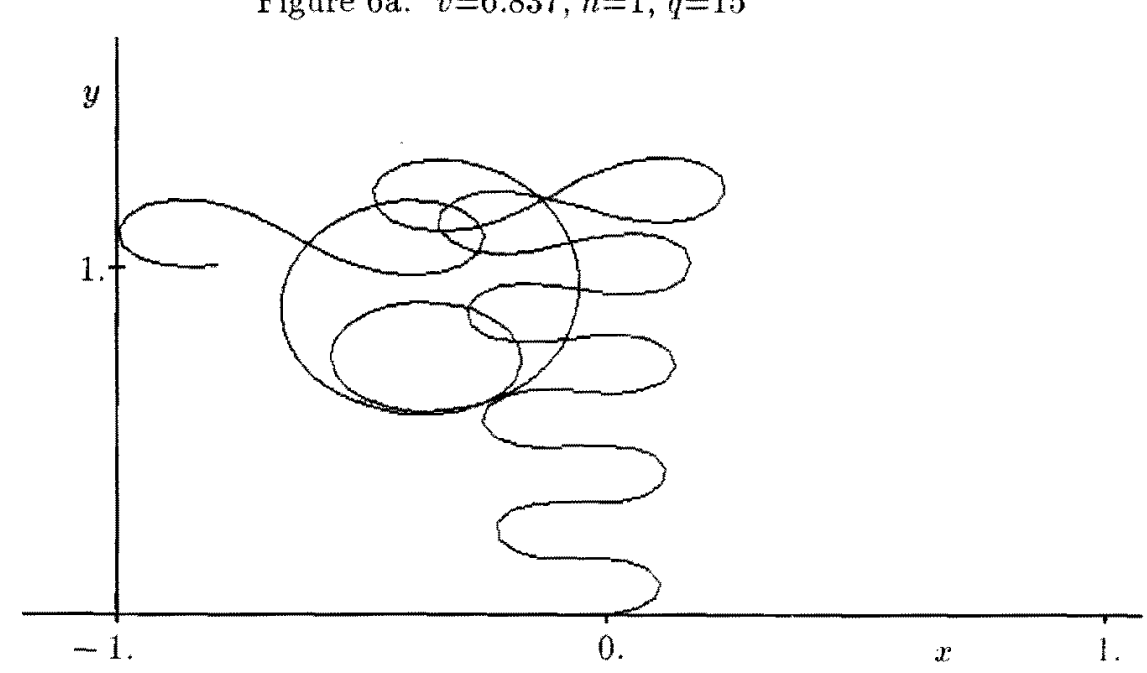

Figure $6 c . \quad v=100, h=1, q=15$

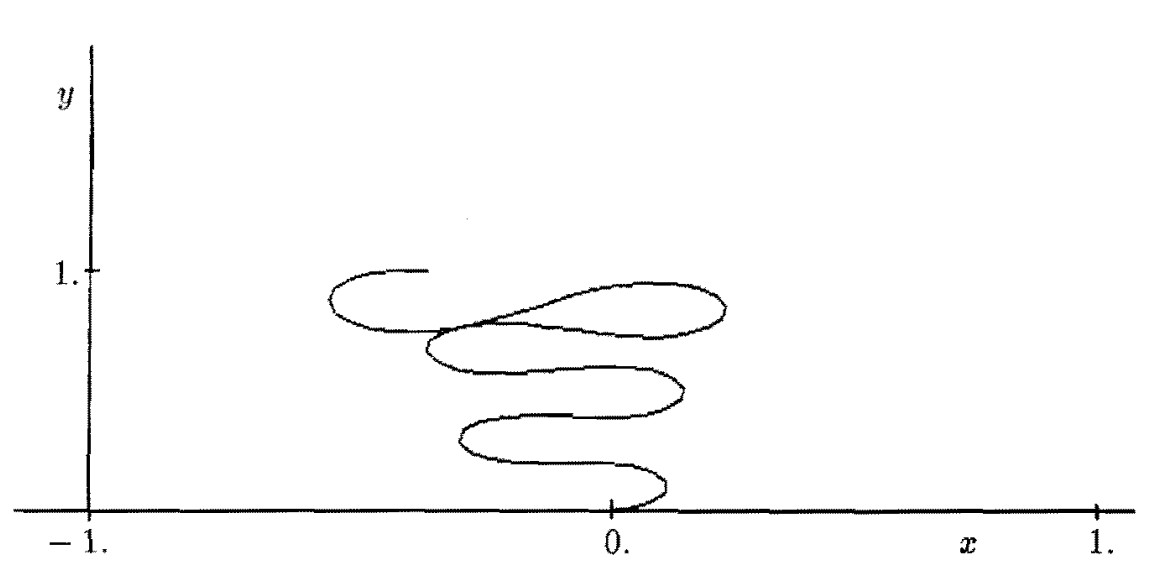

Figure 6b. $v=69, h=1, q=15$

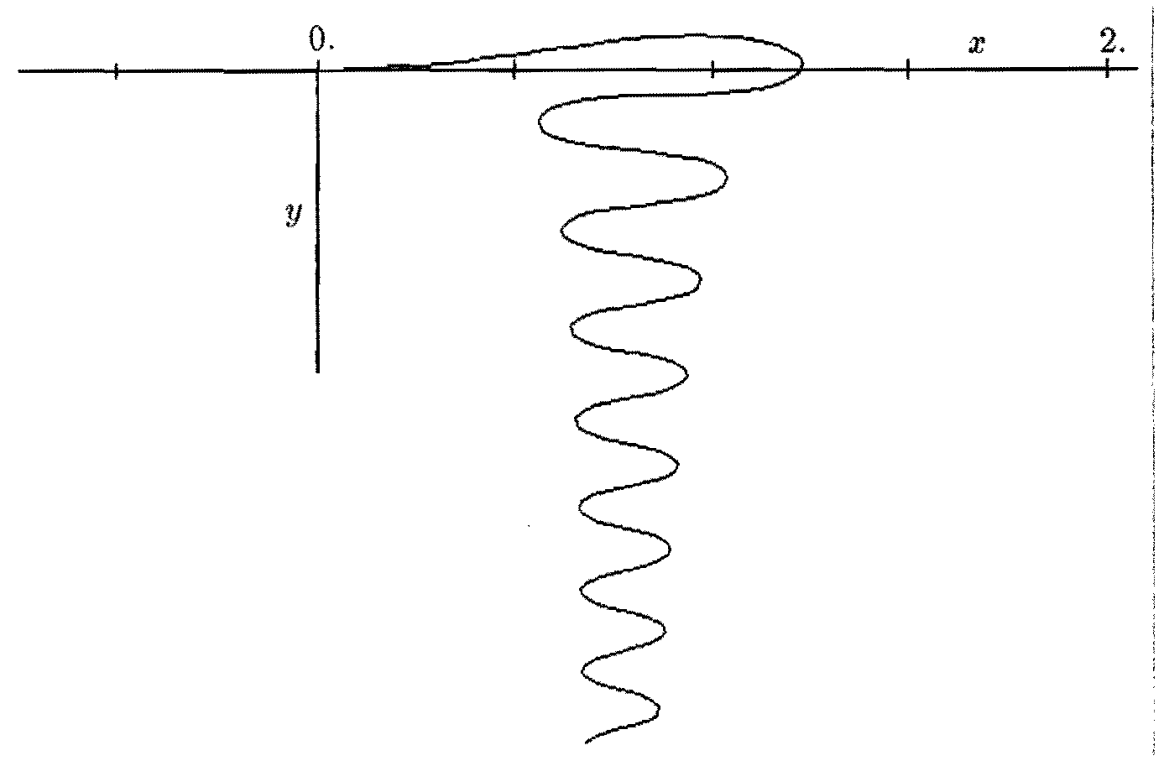

Figure 6d. $v=5, h=1, q=15$ 


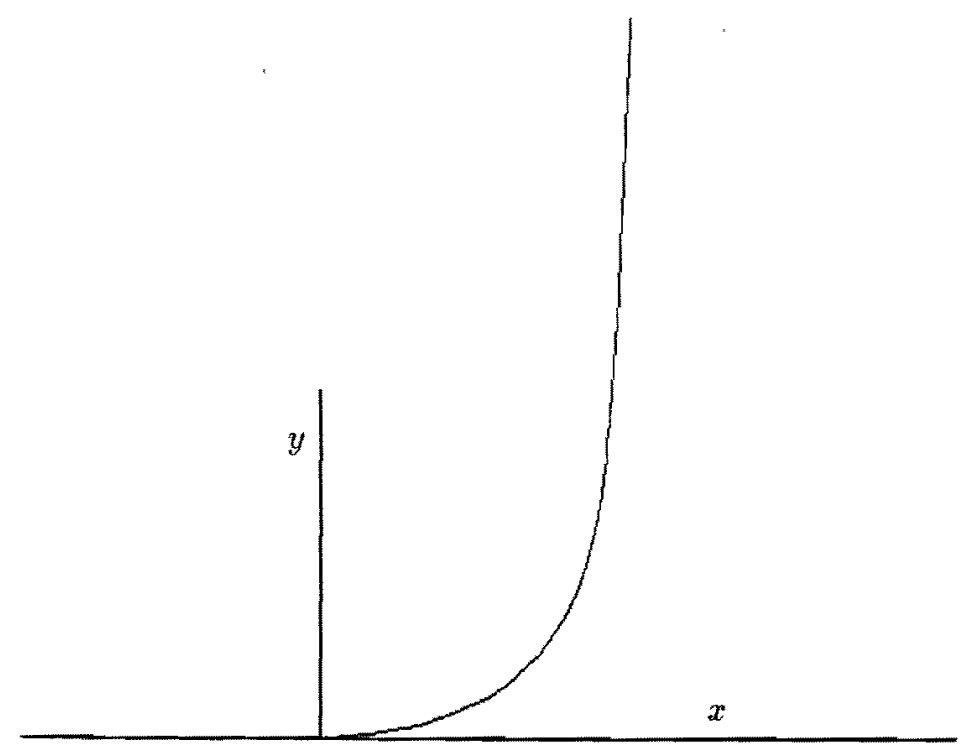

Figure 7a. (First) critical solution in cartesian coordinates $(v=6.837, h=1, q=15)$

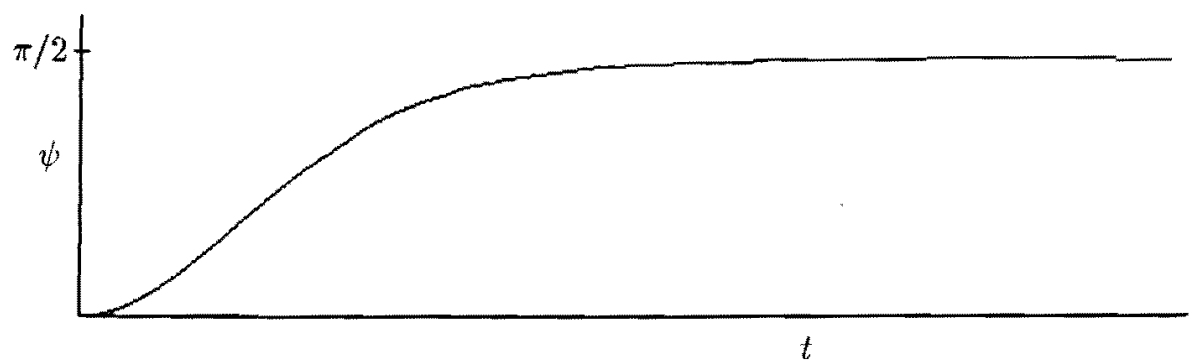

Figure 7b. (First) critical solution in $(\psi, t)$ coordinates $(v=6.837, h=1, q=15)$. 